SCIDiC

\section{Course and Topographic Relationships of Mandibular Canal: A Cone Beam Computed Tomography Study}

ISSN: $2377-8075$

Research Article

Abdallah Edrees MF${ }^{1}$, Moustafa Attia $\mathrm{A}^{2 *}$, Abd Elsattar $\mathrm{MF}^{3}$, Fahmy Gobran $\mathrm{HG}^{4}$, Ismail Ahmed $\mathrm{A}^{5}$

${ }^{1}$ Lecturer of Oral Medicine, Periodontology, Oral Diagnosis and Dental Radiology, Faculty of Dental Medicine - Al-Azhar University (Assiut), Egypt.

${ }^{2}$ Assistant Professor of Oral medicine, Periodontology, Oral Diagnosis and Dental Radiology, Faculty of Dental Medicine - Al-Azhar University

(Assiut) and Umm Alqura Unveristy, Saudi Arabia.

${ }^{3}$ Assistant Lecturer of Oral medicine, Periodontology, Oral Diagnosis and Dental Radiology, Faculty of Dental Medicine - Al-Azhar University (Assiut), Egypt.

${ }^{4}$ Lecturer of Oral Biology, Faculty of Dental Medicine (Cairo), Al-Azhar University, Egypt.

${ }^{5}$ Lecturer of Oral Biology, Faculty of Dental Medicine (Assiut), Al-Azhar University, Egypt.

\title{
Abstract
}

Objectives: The purpose of the present study was to determine the location and configuration of the mandibular canal and related vital structures as a pre-implant assessment using Cone Beam Computed Tomography.

Materials and Methods: 141CBCT scans were undertaken by Kodak $9500^{\circledR}$ Cone Beam 3D Systems and the scanning parameters were $90 \mathrm{kV}, 10 \mathrm{~mA}$, voxel size $0.2 \mathrm{~mm}$ and medium field of view.

Results: The course of the mandibular canal reported (61.17\%) catenary-like, $(28.19 \%)$ progressive descent and $(10.64 \%)$ had a straight pattern. The buccolingual dimension showed that the mandibular canal was located close to the lingual cortical plate (almost $2-3 \mathrm{~mm}$ ) in the molar region and as it proceeds anteriorly it moves toward the buccal aspect of the mandible to emerge finally through the mental foramen. The vertical dimension showed that the canal was located almost $1 \mathrm{~cm}$ above the inferior border of the mandible and then ascended to reach the mental foramen, which is located $15.72 \mathrm{~mm}$ (range 13.82-18.25 $\mathrm{mm}$ ) above the inferior border of the mandible.

Conclusions: Anatomic variations and lesions affecting the mandibular canal were common findings in the CBCT images of the mandible produced for dental implant planning. An awareness of these alterations is important for dentistry because some of them might require treatment, change oral surgery planning and difficult inferior alveolar nerve anesthetic block.

\section{Introduction}

The mandibular canal is a bony canal that emerges from the mandibular foramen on the medial aspect of ramus and runs obliquely in downward and forward direction to ends at the mental foramen. The mandibular canal contains the inferior alveolar artery and the inferior alveolar nerve, which branch to provide innervation to the mandibular teeth and adjacent structures [1].

Identification of the detailed anatomy of the inferior alveolar canal (IAC) including the position, course and morphology is extremely important for dental implantplacement, third molar sur- gery, dental anesthesia, mandibular osteotomy, root canal therapy and any other surgical procedures involving the mandible [2-4].

Many complications, such as altered sensation, paresthesia, numbness and pain, often occurred if vital structures, such as inferior alveolar nerve (IAN) and mental foramen, are not properly identified. In addition, damage of the related blood vessels such as inferior alveolar or lingual artery that induce massive bleeding may also arise from failing to identify these structures. So, it is critical to determine the location and configuration of IAC and related anatomical structures to minimize these types of damages [5].

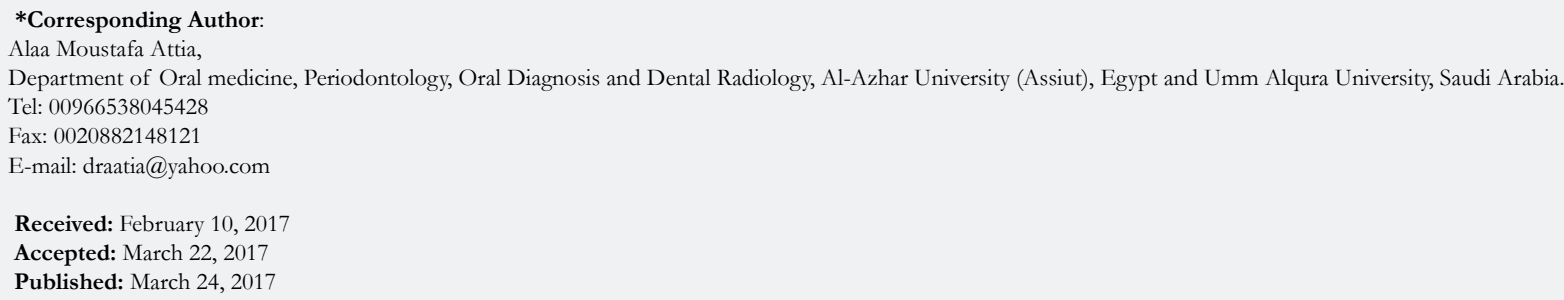

Copyright: Moustafa Attia $\mathbf{A}^{\circ}$ 2017. This is an open-access article distributed under the terms of the Creative Commons Attribution License, which permits unrestricted use, distribution and reproduction in any medium, provided the original author and source are credited. 
Significant limitations have been found in earlier studies describing the intra-bony anatomy of mandibular canal. For example, dry skull reports may have variable diseases and lack relevant data such as age, gender, or include edentulous mandibles, or use inconsistent and anatomically irrelevant landmarks [6].

In particular, the distances between IAC and dental implants or prostheses are so small as to require preoperative evaluation that includes precise measurements with an error of less than 1 to 2 $\mathrm{mm}[7]$.

Panoramic radiograph is a quick, simple, low-dose, and low-cost pre-surgical diagnostic tool for implant planning [8] and provide an excellent general overview of the jaws and the dentition. However, panoramic radiography represents an insufficient method for determining the pre-implant bone status due to magnification in the vertical plane, distortion in the horizontal plane, super-imposition of airway shadows, soft tissue shadows, and ghost images [9] in addition to provision of a $2 \mathrm{D}$ image so the true relationships may not be demonstrated well and neither bucco-lingual width nor angulation of the available bone can be visualized on panoramic radiographs [10].

For many years Computed tomography (CT) has been the gold standard and the most accurate pre-implant evaluation technique, as the information provided is three-dimensional. Its diagnostic value may also be limited by sever dental artifacts, high radiation doses, image noise and higher costs of the procedure [9].

Recently, cone beam computed tomography units has gained considerable importance as a 3D assessment of the dento-maxillofacial region with a single rotation of the gantry. The use of CBCT provides a number of potential advantages over conventional CT, including x-ray beam collimation, reduced effective doses, fewer artifacts and easy handling $[11,12]$.

\section{Materials and Methods}

\section{Study Design and Subjects}

This is a cross-sectional retrospective study carried out on randomly selected 141 CBCT scans (66 male \& 75 female ranged in age from 17 to 70 years with mean age 41.65). These CBCT scans undertaken in the Department of Oral Medicine, Periodontology, Diagnosis, and Oral Radiology, Al-Azhar University between 2012 and 2016 as a part of clinical diagnostic procedure for various reasons, such as implant placement, extraction of the third molar, or orthodontic treatment planning. CBCT scans with positioning errors or subjects with mandibular pathology were excluded from the study. CBCT scans were undertaken by Kodak $9500^{\circledR}$ (Carestream Dental, USA) Cone Beam 3D Systems and the scanning parameters were $90 \mathrm{kV}, 10 \mathrm{~mA}$, voxel size $0.2 \mathrm{~mm}$ and medium field of view.

\section{Image Evaluation}

CBCT images were processed and observed with Blue Sky Plan ${ }^{\circledR}$ (Blue Sky Bio, LLC, Grayslake, IL, USA) program (Version 3.39.5) to assess the following criteria:

1. Course of the mandibular canal:
The course of the mandibular canal, as seen in the panoramic reconstruction, was classified into three types according to Ozturk et al., (2012) [13] and Worthington (2004) [14]: (a) Straight projection, (b) catenary-like configuration; "curled as hanging between two points" and (c) progressive descent.

\section{Location of the mandibular canal in the mandibular body:}

In cross sections of the mandibular arch the following measurements were undertaken:

1. Upper border of the mental foramen (MF) to the inferior and superior border of the mandible.

2. Upper border of the mandibular canal (MC) to the inferior and superior border of the mandible at the level of the mesial root of the first molar.

3. Upper border of the MC to the inferior and superior border of the mandible at the level of the distal root of the first molar.

4. Upper border of the MC to the inferior and superior border of the mandible at the level of the mesial root of the second molar.

5. Upper border of the MC to the inferior and superior border of the mandible at the level of the distal root of the second molar.

6. MC to the buccal and lingual cortex at the level of mesial root of the first molar.

7. MC to the buccal and lingual cortex at the level of distal root of the first molar.

8. MC to the buccal and lingual cortex at the level of mesial root of the second molar.

9. MC to the buccal and lingual cortex at the level of distal root of the second molar.

In panoramic reconstruction, the distance between upper border of the MF to the inferior border of the MC as well as the distance between upper border of the MF to the root tip of $2^{\text {nd }}$ premolar were assessed.

\section{Results}

\section{Course of Mandibular Canal}

Based on our findings the course of the mandibular canal, into 3 different configurations: 1) a straight projection; the final part of the canal was almost at the same level with mental foramen, 2) a catenary-like configuration; "curled as hanging between two points" and 3) progressive descent; the mandibular canal traveled anteriorly and downward, then leveled off around the molar teeth, in almost horizontal course, and then in a sharp ascent to emerge in the mental foramen in the premolar region (Figure 1). The most common configuration was a catenary-like canal present in almost more than one-half of the specimens $(\mathrm{N}=87$; $61.17 \%$ ) without predilection between males and females $(51.7 \%$ to $48.3 \%$ ) and slight predilection $\leq 40$ years by about $55.2 \%$. This pattern was followed by the progressive descent $(\mathrm{N}=39 ; 28.19 \%)$ that showed an obvious predilection in females by about $77 \%$ with slight predilection to found in $\geq 40$ years by about $54 \%$. The remaining specimens $(\mathrm{N}=15 ; 10.64 \%$ ) had a straight pattern that showed a significant predilection in males and $\geq 40$ years by $80 \%$ Table (1). 
Figure 1. Shows the Anatomic Configurations of the Mandibular Canal in the Vertical Plane into Catenary - like (A), Straight (B) and Progressive Descent (C) Respectively.

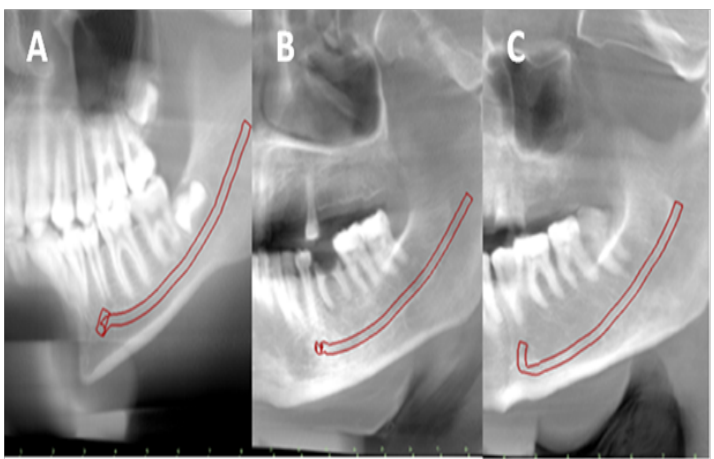

Table 1. Summarize the Anatomic Variations of the Course of Mandibular Canal and its Relations with Gender and Age.

\begin{tabular}{|c|c|c|c|c|c|}
\hline \multicolumn{2}{|c|}{ (141)CBCT scans } & Male (66) & Female (75) & $\leq 40(63)$ & $\geq 40(78)$ \\
\hline Catenary like & (87) $61.17 \%$ & 45 & 42 & 48 & 39 \\
\hline Straight & (15) $10.64 \%$ & 12 & 3 & 3 & 12 \\
\hline Progressive descent & (39) $28.19 \%$ & 9 & 30 & 18 & 21 \\
\hline
\end{tabular}

\section{The Horizontal Location of the MC within the Body of the Mandible}

In the buccolingual dimension, the mandibular canal was found to be either in contact with or close to the lingual cortical plate in the molar region in the majority of cases.

The mean distance from most lingual point of the canal to external surface of lingual cortex at mesial root of $1^{\text {st }}$ molar was $3.67 \pm 1.72$ $\mathrm{mm}$; the mean distance from most lingual point of the canal to external surface of lingual cortex at distal root of $1^{\text {st }}$ molar was $2.24 \pm 1.43$ $\mathrm{mm}$; the mean distance from most lingual point of the canal to external surface of lingual cortex at mesial root of $2^{\text {nd }}$ molar was $2.15 \pm 1.30$ $\mathrm{mm}$; and the mean distance from most lingual point of the canal to external surface of lingual cortex at distal root of $2^{\text {nd }}$ molar was $2.13 \pm$ $1.26 \mathrm{~mm}$ (Figure 2, Table 2).

The mean distance from most buccal point of the canal to external surface of buccal cortex at mesial root of $1^{\text {st }}$ molar was $4.09 \pm 1.54 \mathrm{~mm}$; the mean distance from most buccal point of the canal to external surface of buccal cortex at distal root of 1 stmolar was $5.34 \pm 1.80 \mathrm{~mm}$; the mean distance from most buccal point of the canal to external surface of buccal cortex at mesial root of $2^{\text {nd }}$ molar was $6.04 \pm 1.76 \mathrm{~mm}$; and the mean distance from most buccal point of the canal to external surface of buccal cortex at distal root of $2^{\text {nd }}$ molar was $6.25 \pm 2.00$ $\mathrm{mm}$ (Figure 2, Table 2).

The vertical location of the MC within the body of the mandible

In vertical dimension, the detailed anatomy of the mandibular canal was studied considering the inferior and superior borders of the mandible as reference points. The measurements of the upper border of the mandibular canal with respect to the body of the mandible were performed at 5 different points (mental foramen, mesial and distal roots of first and second molars).

The course of MC in relation to the inferior border of the mandible: The mean vertical position as measured from the upper border of the mandibular canal to the inferior border of the mandible at the level of the mesial root of 1 st molar was 11.39 $\pm 2.03 \mathrm{~mm}$, ranging from 9.70 to $14.46 \mathrm{~mm}$. The mean vertical position as measured from the upper border of the mandibular canal to the inferior border of the mandible at the level of the distal root of 1 st molar was $10.81 \pm 1.75 \mathrm{~mm}$, ranging from 9.65 to $13.80 \mathrm{~mm}$. The mean vertical position as measured from the upper border of the mandibular canal to the inferior border of the mandible at the level of the mesial root of $2^{\text {nd }}$ molar was 10.86 $\pm 2.21 \mathrm{~mm}$, ranging from 8.94 to $14.70 \mathrm{~mm}$. The mean vertical position as measured from the upper border of the mandibular canal to the inferior border of the mandible at the level of the distal root of $2^{\text {nd }}$ molar was $11.33 \pm 2.30 \mathrm{~mm}$, ranging from 8.87 to $15.40 \mathrm{~mm}$ (Figure 3, Table 2).

The course of MC in relation to the superior border of the mandible: The mean vertical position as measured from the upper border of the mandibular canal to the superior border of the mandible at the level of the mesial root of $1^{\text {st }}$ molar was 18.38 $\pm 2.04 \mathrm{~mm}$, ranging from 15.00 to $21.01 \mathrm{~mm}$. The mean vertical position as measured from the upper border of the mandibular canal to the superior border of the mandible at the level of the distal root of $1^{\text {st }}$ molar was $17.63 \pm 1.81 \mathrm{~mm}$, ranging from 15.10 to $20.90 \mathrm{~mm}$. The mean vertical position as measured from the upper border of the mandibular canal to the superior border of the mandible at the level of the mesial root of $2^{\text {nd }}$ molar was 17.43 $\pm 2.20 \mathrm{~mm}$, ranging from 15.10 to $21.23 \mathrm{~mm}$. The mean vertical position as measured from the upper border of the mandibular canal to the superior border of the mandible at the level of the distal root of $2^{\text {nd }}$ molar was $17.29 \pm 2.69 \mathrm{~mm}$, ranging from 12.30 to $20.90 \mathrm{~mm}$ (Figure 3, Table 2).

\section{The Relations of MF}

The mean vertical position as measured from the upper border of the mental foramen to the inferior border of the mandible was $15.72 \pm 1.84 \mathrm{~mm}$, ranging from 13.82 to $18.25 \mathrm{~mm}$. The mean vertical position as measured from the upper border of MF to 
Figure 2. Shows Relations of Mandibular Canal to the Buccal and Lingual Cortex at the Level of Mesial and Distal Root of $1^{\text {st }}$ and $2^{\text {nd }}$ Molar Respectively.

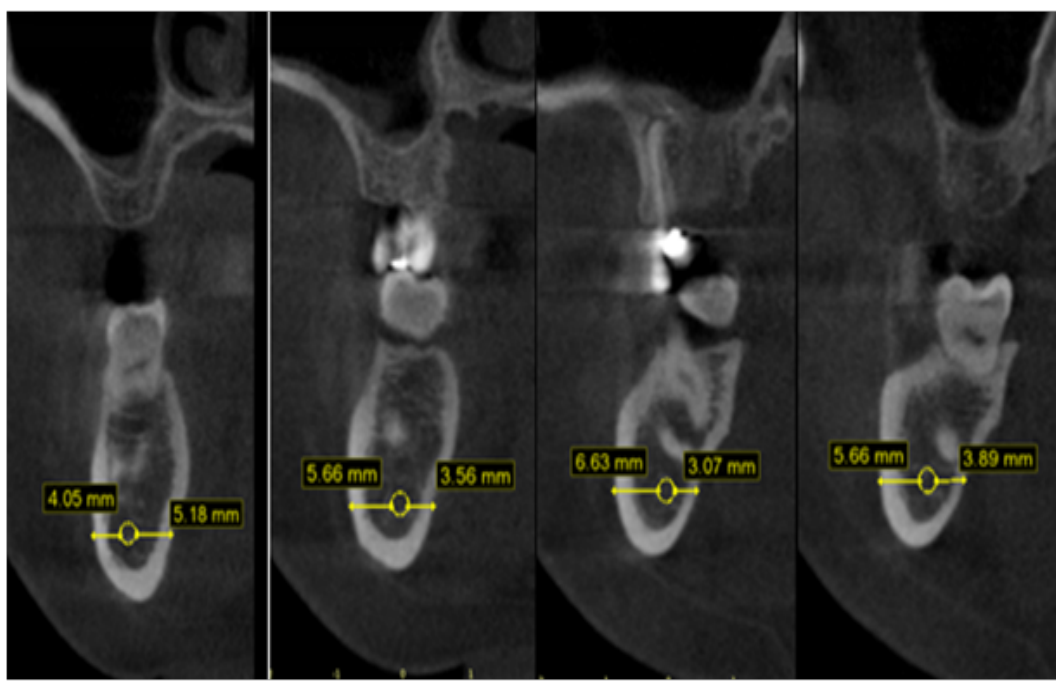

Table 2. Summarize mean, standard deviation, minimum and maximum values of all cone-beam CT measurements; UD, upper distance: the distance between upper border of $\mathrm{MC}$ and the superior border of the alveolar ridge; $\mathrm{LD}$, lower distance: the distance between upper border of MC and the inferior border of the mandible; BD, buccal distance: the distance between the most buccal point of the canal and the perpendicular point on the buccal margin of the mandible; LID, lingual distance: the distance between the most lingual point of the canal and the perpendicular point on the lingual margin of the mandible.

\begin{tabular}{|c|c|c|c|c|c|}
\hline MC & & UD & LD & BD & LID \\
\hline \multirow{4}{*}{ Mesial root $1^{\text {st }}$ molar } & Mean & 18.38 & 11.39 & 4.09 & 3.67 \\
\hline & $\mathrm{SD} \pm$ & 2.04 & 2.03 & 1.54 & 1.72 \\
\hline & Min & 15.00 & 9.70 & 2.17 & 1.51 \\
\hline & $\operatorname{Max}$ & 21.01 & 14.46 & 5.82 & 6.74 \\
\hline \multirow{4}{*}{ Distal root $1^{\text {st }}$ molar } & Mean & 17.63 & 10.81 & 5.34 & 2.24 \\
\hline & $\mathrm{SD} \pm$ & 1.81 & 1.75 & 1.80 & 1.43 \\
\hline & Min & 15.10 & 9.65 & 2.38 & 0.95 \\
\hline & Max & 20.90 & 13.80 & 8.19 & 4.68 \\
\hline \multirow{4}{*}{ Mesial root $2^{\text {nd }}$ molar } & Mean & 17.43 & 10.86 & 6.04 & 2.15 \\
\hline & $\mathrm{SD} \pm$ & 2.20 & 2.21 & 1.76 & 1.30 \\
\hline & Min & 15.10 & 8.94 & 3.15 & 0.61 \\
\hline & $\operatorname{Max}$ & 21.23 & 14.70 & 8.37 & 4.20 \\
\hline \multirow{4}{*}{ Distal root $2^{\text {nd }}$ molar } & Mean & 17.29 & 11.33 & 6.25 & 2.13 \\
\hline & $\mathrm{SD} \pm$ & 2.69 & 2.30 & 2.00 & 1.26 \\
\hline & Min & 12.30 & 8.87 & 3.19 & 0.43 \\
\hline & $\operatorname{Max}$ & 20.90 & 15.40 & 9.36 & 3.96 \\
\hline
\end{tabular}

upper border of the mandible was $15.41 \pm 1.85 \mathrm{~mm}$, ranging from 13.00 to $18.86 \mathrm{~mm}$. The mean vertical position as measured from the upper border of MF to inferior border of MC was $4.21 \pm 1.23$ $\mathrm{mm}$, ranging from 2.80 to $5.90 \mathrm{~mm}$. The mean vertical position as measured from the upper border of MF to the root tip of 2nd premolar was $4.41 \pm 2.49 \mathrm{~mm}$, ranging from 1.20 to $5.70 \mathrm{~mm}$ (Figure 4, Table 3).

\section{Discussion}

Presently, CBCT and Multi Slice Computed Tomography (MSCT) have been recommended as appropriate diagnostic imaging tools for assessment of dental implant; the accuracy of both CBCT and MSCT images is reportedly high in linear measurement [15]. In addition; the diagnostic quality and depiction of fine anatomic features in the mandible associated with neurovascular structures is consistent between CBCT and MSCT images [16]. The present study used CBCT due to its superiority over MSCT as; the radiation dose is relatively low, CBCT machine can be used easily and effectively in a dental clinic and the higher level of resolution in CBCT images than that in MSCT images [17].

The course of the mandibular canal axial and sagittal views was studied in CBCT images. The mandibular canal followed 3 differ- 
Figure 3. Shows Relations of Upper Border of Mandibular Canal to the Superior and Inferior Border of the Mandible at the Level of $1^{\text {st }}$ and $2^{\text {nd }}$ Molar Respectively.

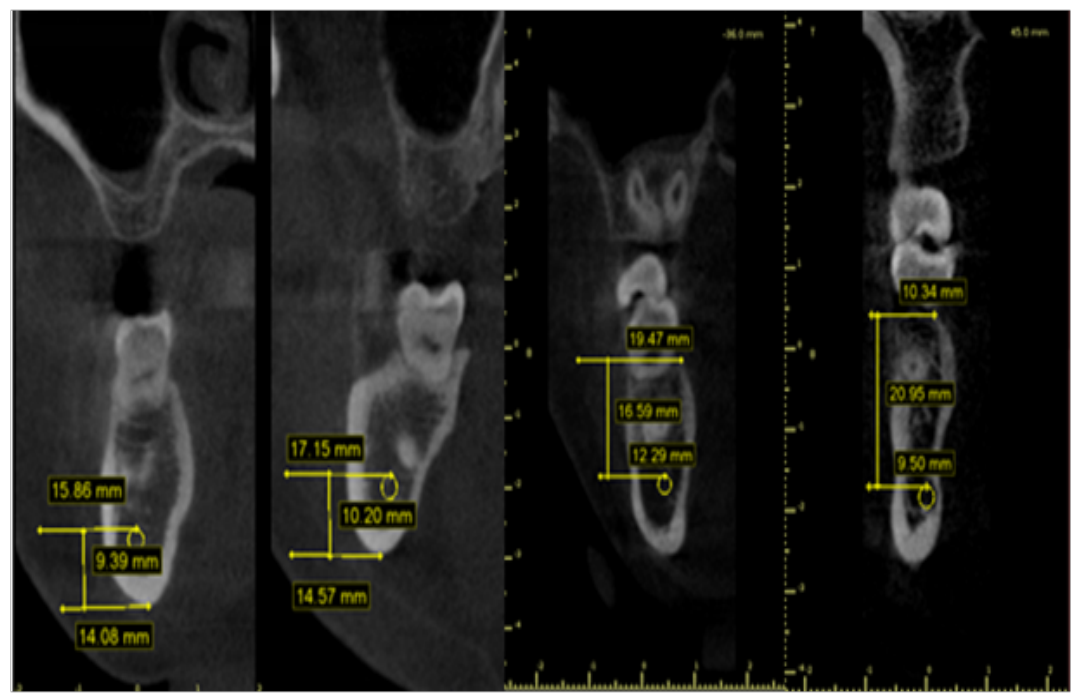

Figure 4. Shows Relations of Upper Border of Mental Foramen to Inferior Border of Both the Mandible and Mandibular Canal Respectively.

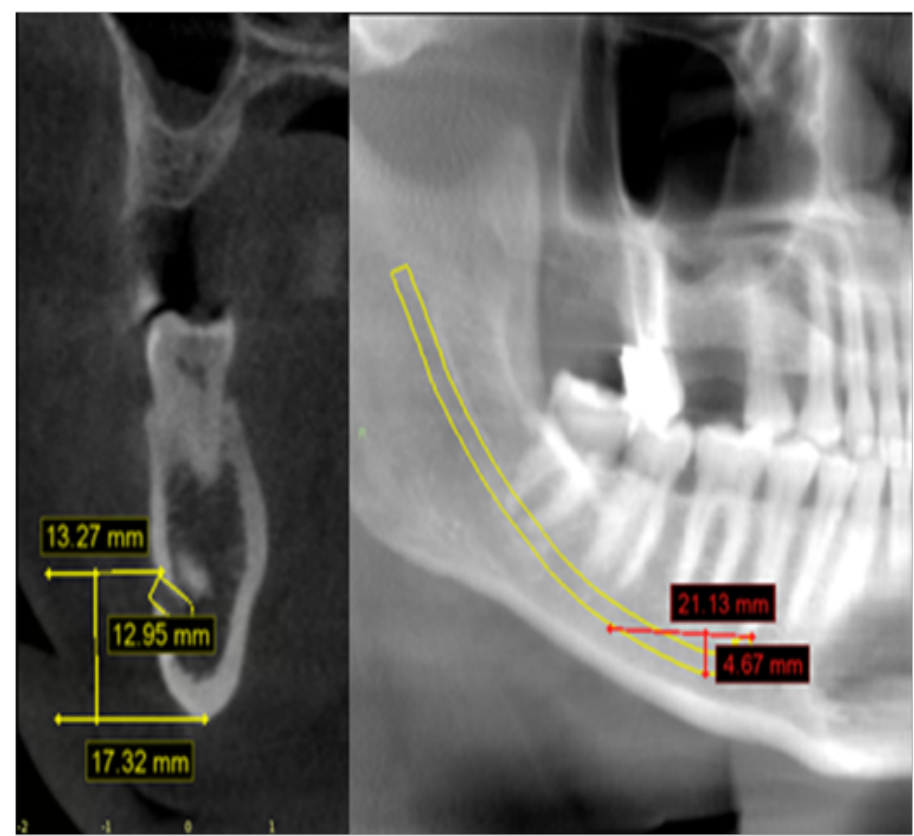

Table 3. Summarize Mean, Standard Deviation, Minimum and Maximum Distance from the Upper Border of MF to Superior (UD) and Inferior border (LD) of the Mandible as well as to Root tip of $2^{\text {nd }}$ Premolar and the Inferior Border of MC.

\begin{tabular}{|c|c|c|c|c|c|}
\hline \multicolumn{2}{|c|}{} & UD & LD & UB to $2^{\text {nd }}$ premolar root tip & UB of MF to LB of MC \\
\hline \multirow{3}{*}{ MF } & Mean & 15.41 & 15.72 & 4.41 & 4.21 \\
\cline { 2 - 6 } & SD \pm & 1.85 & 1.84 & 2.49 & 1.23 \\
\cline { 2 - 6 } & Min & 13.00 & 13.82 & 1.20 & 2.80 \\
\cline { 2 - 6 } & Max & 18.86 & 18.25 & 5.70 & 5.90 \\
\hline
\end{tabular}

ent patterns: a straight projection, a catenary-like configuration and progressive descent. In our study, only a small percentage $(10.64 \%)$ had the mandibular canal fitting the classic description of straight projection. This group is less favorable for implant placement posterior to premolars. The most common pattern found in our study group was the catenary-like canal $(61.17 \%)$. This pattern provides more space for implant placement, espe- cially in the first molar region compared with the premolar region. This was followed by the progressive descent $(28.19 \%)$. The findings of this study are nearly in agreement with the results of $\mathrm{Oz}-$ turk et al., (2012) [13] on adult dry skulls who recorded (51.1\%) catenary-like, $(36.7 \%)$ progressive descent and $(12.2 \%)$ straight projection and very similar to a recent report on panaromic radiographs by Liu et al., (2009) [18]. 
It was showed that, as the MC runs from the mandibular foramen obliquely not only in downward and forward direction, but also in the buccolingual dimension, it become more buccally within the body of the mandible as it emerges at the mental foramen i.e. in the molar area, the mandibular canal was either in contact with the lingual cortex or ran very close to the lingual cortex while in the premolar area, the mental nerve usually makes a sharp turn from lingual to buccal and exits through the mental foramen. Similar courses have been recorded by several other investigators $[13,19-21]$. The buccolingual course of the MC becomes more important in cases of deficiency of the vertical dimension of the alveolar ridge. Kim et al., (2009) [21] suggested that implants may be placed buccal to the mandibular canal with minor adjustment of angulation when itscourse runs close to the lingual cortex.

As the distance from the $\mathrm{MC}$ to the superior border of the mandible is very important in adequate implant length selection, it has less significance and was greater variability (range $21.23-12.30 \mathrm{~mm}$ ) may be due to bone resorption. Therefore, the inferior border of the body of the mandible seems to be a more predictable reference. In the vertical dimension, on average, the mandibular canal ran almost $1 \mathrm{~cm}$ above the inferior border of the body of the mandible in the molar region. Our results are in agreement with 2 cadaveric studies where the inferior alveolar nerve was found to be situated $\sim 1 \mathrm{~cm}$ above the mandibular inferior border $[22,23]$.

The mean distances between the upper border of the mental foramen and the inferior border of the mandible was $15.72 \mathrm{~mm}$. This is in accordance with earlier study reporting 14.7and $14.61 \mathrm{~mm}$ [24]. The $1 \mathrm{~mm}$ difference may result from different reference points at MF.

The present study reported a strong correlation between the vertical position of the mental foramen from the inferior border of mandible and vertical length of the mandible at the premolar region; the higher the vertical length, the higher the location of the mental foramen.

\section{References}

[1]. Greenstein G, Cavallaro J, Tarnow D (2008) Practical application of anatomy for the dental implant surgeon. J Periodontol. 79(10): 1833-1846.

[2]. Hillerup S, Jensen R (2006) Nerve injury caused by mandibular block analgesia. Int J Oral Maxillofac Surg. 35(5): 437-443.

[3]. Escoda-Francoli J, Canalda-Sahli C, Soler A, Figueiredo R, Gay-Escoda C (2007) Inferior alveolar nerve damage because of overextended endodontic material: a problem of sealer cement biocompatibility? J Endodontics. 33(12): 1484-1489.

[4]. Gz Xu, Yang C, Fan XD, Yu CQ, He D, et al., (2013) Anatomic relationship between impacted third mandibular molar and the mandibular canal as the risk factor of inferior alveolar nerve injury. Br J Oral Maxillofac Surg.
51(8): e215-e219.

[5]. Kim IS, Kim SG, Kim YK, Kim JD (2006) Position of the mental foramen in a Korean population: a clinical and radiographic study. Implant Dent. 15(4): 404-11

[6]. Hwang K, Lee WJ, Song YB, Chung IH (2005) Vulnerability of the inferior alveolar nerve and mental nerve during genioplasty: an anatomic study. J Craniofac Surg. 16(1): 10-14.

[7]. Feifel H, Riediger D, Gustorf-Aeckerle R (1994) High resolution computed tomography of the inferior alveolar and lingual nerves. Neuroradiology. 36(3): 236-8.

[8]. Vazquez L, Saulacic N, Belser U, Bernard JP (2008) Efficacy of panoramic radiographs in the preoperative planning of posterior mandibular implants: a prospective clinical study of 1527 consecutively treated patients. Clin Oral Implants Res. 19(1): 81-85.

[9]. Mansour P, Dudhia R (2008) Implant radiography and radiology. Aust Dent J. 53(1): 511-525.

[10]. Garg AK (2007) Dental implant imaging: TeraRecon's dental 3D cone beam computed tomography system. Dent Implantol Update. 18(6): 41-45.

[11]. Scarfe WC, Farman AG, Sukovic P (2006) Clinical applications of conebeam computed tomography in dental practice. J Can Dent Assoc. 72(1): 75-80.

[12]. Gupta J, Ali SP (2013) Cone beam computed tomography in oral implants. N J Maxillofac Surg. 4(1): 2-6.

[13]. Ozturk, Potluri, Vieira (2012) Position and course of the mandibular canal in skulls. Oral Surgery Oral Medicine Oral Pathology Oral Radiol. 113(4): 453-458.

[14]. Worthington P (2004) Injury to the inferior alveolar nerve during implant placement: a formula for protection of the patient and clinician. Int J Oral Maxillofac Implant. 19(5): 731-4.

[15]. Ito K, Gomi Y, Sato S, Arai Y, Shinoda K (2001) Clinical application of a new compact CT system to assess 3-D images for the preoperative treatment planning of implants in the posterior mandible: a case report. Clin Oral Implants Res. 12(5): 539-42.

[16]. Dreiseidler T, Mischkowski RA, Neugebauer J, Ritter L, ZoellerVJE (2009) Comparison of cone-beam imaging with orthopantomography and computerized tomography for assessment in presurgical implant dentistry. Int J Oral Maxillofacial Implants. 24(2): 216-25.

[17]. Naitoh M, Nakahara K, Suenaga Y, Gotoh K, Kondo S (2010) Comparison between cone-beam and multislice computed tomography depicting mandibular neurovascular canal structures. Oral Surgery Oral Med Oral Pathol Oral Radiol. 109: e25-e31.

[18]. Liu T, Xia B, Gu Z (2009) Inferior alveolar canal course: a radiographic study. Clin Oral Implants Res. 20(11): 1212-8.

[19]. Hwang K, Lee WJ, Song YB, Chung IH (2005) Vulnerability of the inferior alveolar nerve and mental nerve during genioplasty: an anatomic study. J Craniofac Surg. 16(1): 10-4.

[20]. Jung YH, Nah KS, Cho BH (2007) The location of the mandibular canal in prognathic patients compared to subjects with normal occlusion. Korean J Oral Maxillofaci Radiol. 37(4): 217-20.

[21]. Kim ST, Hu KS, Song WC, Kang MK, Park HD, et al., (2009) Location of the mandibular canal and the topography of its neurovascular structures. Journal of Craniofacial Surg. 20(3): 936-9.

[22]. Rajchel J, Ellis E third, Fonseca RJ (1986) The anatomical location of the mandibular canal: its relationship to the sagittal ramus osteotomy. Int J Adult Orthodon Orthognathic Surg. 1(1): 37-47.

[23]. Mbajiorgu EF, Mawera G, Asala SA, Zivanovic S (1998) Position of the mental foramen in adult black Zimbabwean mandibles: a clinical anatomical study. Cent Afr J Med. 44(2): 24-30.

[24]. Oguz O, Bozkir MG (2002) Evaluation of location of mandibular and mental foramina in dry, young, adult human male, dentulous mandibles. West Indian Med J. 51(1): 14-6. 